\author{
Artículo original \\ (Original paper)
}

\title{
SCOLYTINAE Y PLATYPODINAE (COLEOPTERA: CURCULIONIDAE) DE TABASCO, MÉXICO
}

\author{
SCOLYTINAE AND PLATYPODINAE (COLEOPTERA: CURCULIONIDAE) OF TABASCO, \\ MEXICO
}

\section{ARMANDo FALCÓN-BRINDIS ${ }^{1}$, MANUEl PÉREZ-DE LA CRUZ ${ }^{1}{ }^{*}$, ENA EdTTH MATA-ZAYAS ${ }^{1}$, ARACELY DE LA CRUZ-PÉREZ ${ }^{1}$, SAÚL SÁNCHEZ-SOTO ${ }^{2}$ y CARLOS M. BURELO-RAMOS ${ }^{1}$}

\footnotetext{
${ }^{1}$ Universidad Juárez Autónoma de Tabasco, División Académica de Ciencias Biológicas, C.P. 86150, Carretera VillahermosaCárdenas km 0.5 s/n entronque a Bosque de Saloya, Villahermosa, Tabasco, México. <armandofalcon14@hotmail.com>; <perezmandoc@hotmail.com>; <ena.matazayas@gmail.com>; <arace_lycp@hotmail.com>; <carlos.burelo@ujat.mx>.

${ }^{2}$ Colegio de Postgraduados, Campus Tabasco, Apartado postal 24, C.P. 86500, H. Cárdenas Tabasco, México. <sssoto@colpos.mx>

* Autor para correspondencia: <perezmandoc@hotmail.com>
}

Recibido: 29/06/2017; aceptado: 05/03/2018; publicado en línea: 23/10/2018

Editor responsable: Arturo Bonet Ceballos

Falcón-Brindis, A., Pérez de la Cruz, M., Mata-Zayas, E. E., De la Cruz-Pérez, A., Sánchez-Soto, S. y Burelo-Ramos, C. M. (2018). Scolytinae y Platypodinae (Coleoptera: Curculiondae) de Tabasco, México. Acta Zoológica Mexicana (nueva serie), 34(2), 1-10. DOI: 10.21829/azm.2018.3412107

RESUMEN. El objetivo del presente estudio fue evaluar la diversidad de Scolytinae y Platypodinae en cinco localidades de Tabasco de diciembre de 2012 y abril de 2013. Se utilizaron diez trampas de intercepción de vuelo con alcohol etílico como atrayente para recolectar a los insectos en cada sitio. Se recolectaron en total 4,232 especímenes de 62 especies, de las cuales 57 pertenecen a Scolytinae y cinco a Platypodinae. La mayor diversidad de especies se obtuvo en Malpasito (MP) con un valor de $\mathrm{H}^{\prime}=2.11$ y la menor en Boca del cerro (BC) con un valor de $\mathrm{H}^{\prime}=0.78$, la mayor riqueza de especies la registró MP con 39 y la mayor abundancia Francisco Rueda (FR) con 1,500 especímenes. Los géneros Hypothenemus y Xyleborus presentaron la mayor riqueza de especies con diez y siete respectivamente. Las especies Premnobius cavipennis Eichhoff, Xyleborus volvulus (Fabricius), Sampsonius dampfi Schedl, Hypothenemus interstitialis (Hopkins) y Corthylus papulans Eichhoff registraron la mayor abundancia con 2,962 especímenes, lo que representa el 69.99\% del total. Las especies Cnesinus elegans Blandford, C. gracilis Blandford, Corthylocurus barbatus (Blandford), Chramesus crenatus Wood, H. effeminatus Wood, $H$. inaequalis Wood, $H$. columbi Hopkins y $M$. robustum (Schedl) son nuevos registros para Tabasco. La lista de especies de estos insectos en Tabasco, México continúa creciendo conforme se incrementan los muestreos, lo que sugiere que no ha sido posible recolectar la diversidad real que albergan los ecosistemas del estado.

Palabras clave: insectos; barrenadores; ambrosia; tropicales 
Falcón-Brindis, A., Pérez de la Cruz, M., Mata-Zayas, E. E., De la Cruz-Pérez, A., Sánchez-Soto, S. \& Burelo- Ramos, C. M. (2018). Scolytinae and Platypodinae (Coleoptera: Curculionidae) of Tabasco, Mexico. Acta Zoológica Mexicana (n.s.), 34(2), 1-10. DOI: 10.21829/azm.2018.3412107

\begin{abstract}
The aim of the present study was to evaluate the diversity of Scolytinae and Platypodinae in five localities of Tabasco between December 2012 and April 2013. Ten flight intercept traps were used with ethyl alcohol as an attractant to collect insects in each site. A total of 4,232 specimens were collected from 62 species, of which 57 belong to Scolytinae and five to Platypodinae. The greatest diversity of species was obtained in Malpasito (MP) with a value of $\mathrm{H}^{\prime}=2.11$ and the lowest in Boca del Cerro (BC) with a value of $\mathrm{H}^{\prime}=0.78$, the highest species richness was recorded MP with 39 and the highest species abundance Francisco Rueda (FR) with 1,500 specimens. The genera Hypothenemus and Xyleborus presented the highest species richness with 10 and 7 respectively. The species Premnobius cavipennis Eichhoff, Xyleborus volvulus (Fabricius), Sampsonius dampfi Schedl, Hypothenemus interstitialis (Hopkins) and Corthylus papulans Eichhoff showed the highest abundance with 2,962 specimens, representing $69.99 \%$ of the total. The especies Cnesinus elegans Blandford, C. gracilis Blandford, Corthylocurus barbatus (Blandford), Chramesus crenatus Wood, $H$. effeminatus Wood, $H$. inaequalis Wood, $H$. columbi Hopkins and $M$. robustum (Schedl) are new records for Tabasco. The list of species of these insects in Tabasco, México continues to grow as new samples are taken, suggesting that it has not been possible to collect the real diversity supported by the ecosystems of the state.
\end{abstract}

Key words: insects; borers; ambrosia; tropical

\title{
INTRODUCCIÓN
}

Scolytinae y Platypodinae son escarabajos que se alimentan y reproducen en plantas recién muertas, decadentes o vivas, realizan galerías que a menudo interrumpen el flujo de savia en su huésped, lo que puede afectar el rendimiento o incluso ocasionarle la muerte (Wood \& Bright, 1992a; 1992b; Logan \& Powell, 2001). Estos insectos, se alimentan de floema (escarabajos de la corteza) u hongos ectosimbióticos que cultivan en sus túneles (escarabajos de ambrosia), pueden atacar xilema, semillas, frutos y plantas herbáceas (Wood, 1982; 1993). En el mundo, un gran número de especies barrenadoras de madera se están estableciendo continuamente en nuevas áreas, lo que ha generado preocupación en las regiones con importantes recursos forestales (Haack, 2006; Kirkendall \& Facolli, 2010). En las zonas tropicales, estos escarabajos pueden alcanzar niveles importantes en sus poblaciones bajo condiciones de alta humedad, lo cual permite el establecimiento de hongos ectosimbióticos (Kirkendall et al., 2014). En Tabasco, actualmente se tiene registrado 93 especies de Scolytinae y 7 especies de Platypodinae recolectados en diferentes tipos de vegetación, tales como; selvas, manglares, plantaciones de cacao (agroecosistemas) y vegetación secundaria, sin embargo, se ha logrado observar que la lista de especies continúa creciendo conforme se realizan nuevos muestreos, lo que hace pensar que no se ha logrado recolectar la diversidad real que albergan los ecosistemas de Tabasco (Pérez-De La Cruz et al., 2009; 2011; 2015; 2016; Gerónimo-Torres et al., 2015). Por tal motivo, el objetivo del presente estudio fue determinar la diversidad de especies de Scolytinae y Platypodinae en cinco sitios de Tabasco que no habían sido abordado en estudios anteriores, con la finalidad de contribuir al conocimiento que resguardan nuestros ecosistemas tropicales.

\section{MATERIALES Y MÉTODOS}

El estudio se realizó en cinco localidades de Tabasco, México. Las recolectas se llevaron a cabo de diciembre de 2012 a abril de 2013, época en la que estos insectos registran su mayor actividad (Pérez-De 
la Cruz, 2009) en: Boca del Cerro (BC), el muestreo se realizó en el ejido Lázaro Cárdenas, municipio de Tenosique, el cual pertenece al Parque Estatal Cañón del Usumacinta localizada a $17^{\circ} 23^{\prime} 45^{\prime}$ N y 91²3’51”W; en esta zona se encuentran elevaciones máximas de hasta $300 \mathrm{~m}$ snm con vegetación de selva alta perennifolia (INEGI, 2000; 2001; Guzmán \& Bello, 2006). El segundo sitio se localiza en la zona aledaña a la Zona Arqueológica Malpasito (MP), perteneciente a la Reserva Ecológica de Agua Selva, municipio de Huimanguillo, Tabasco, la cual ocupa un área de 10,790.28 ha, ubicada a $17^{\circ} 19^{\prime} 81^{\prime}$ 'N y 93³3'54' 'W, con altitudes que van desde 200 a $1000 \mathrm{~m}$ snm con vegetación de selva alta perennifolia. El tercer sitio pertenece a la Ranchería Campo Alto (CA) municipio de Balancán ubicado a $17^{\circ} 52^{\prime} 21^{\prime} \mathrm{N}$ y $91^{\circ} 29^{\prime} 05^{\prime}$ 'W, en este sitio predomina el pastizal cultivado, sin embargo, en la periferia se encuentra un fragmento de vegetación conservada con presencia de especies arbóreas que alcanzan los 10 $\mathrm{m}$ de altura. El cuarto sitio pertenece a la Ranchería Ribera Alta (RA) municipio de Centla, se localiza a $18^{\circ} 20^{\prime} 46^{\prime} \mathrm{N}$ y $92^{\circ} 32^{\prime} 03^{\prime} \mathrm{W}$, la vegetación predominante es pastizal inundable, aledaña a esta área se encontró vegetación de galería, popal y tular, las cuales son representativas de las zonas bajas inundables del estado. El último sitio fue Francisco Rueda (FR), una plantación de hule (Hevea brasiliensis Mull. Arg.), municipio de Huimanguillo, localizado a $17^{\circ} 48^{\prime} 20^{\prime} \mathrm{N}$ y $93^{\circ} 37^{\prime} 19^{\prime \prime} \mathrm{W}$. La vegetación predominante es sabana con lomeríos que no sobrepasan los $30 \mathrm{~m}$ snm. Los sitios presentan un clima cálido húmedo con abundantes lluvias en verano y cálido húmedo con lluvias todo el año. La precipitación promedio es de 2,500 mm/año (INEGI, 2013).

Captura de insectos. Se colocaron 10 trampas de intercepción de vuelo, cebadas con alcohol etílico como atrayente, en cada una de las localidades estudiadas a una altura de $1.50 \mathrm{~m}$, y $100 \mathrm{~m}$ de distancia entre trampa. La recolección de los insectos se realizó a los 15 días posteriores a su colocación en cada sitio, los especímenes se conservaron en alcohol al 70\% para su posterior montaje e identificación en el laboratorio de la colección de insectos de la División Académica de Ciencias Biológicas (CIUT) (Bustamante \& Atkinson, 1984; Iturre \& Darchuck, 1996; Pérez-De La Cruz et al., 2009).

Identificación del material biológico. El montaje y etiquetado de los insectos se realizó de acuerdo a la metodología de Triplehorn \& Johnson (2005) y la determinación taxonómica de los insectos se realizó usando las claves taxonómicas de Wood (1982; 1993) y Pérez-De La Cruz et al. (2011).

Análisis de diversidad. Para comparar la diversidad de Scolytinae y Platypodinae se utilizaron los índices de diversidad de Shannon-Wiener $\left(\mathrm{H}^{\prime}\right)$, que mide la estructura de la comunidad; riqueza de Margalef (Dmg), el cual registra la riqueza específica; el índice de Pielou (J), el cual toma en cuenta la equidad de la comunidad y el índice de similitud de Sorensen (Is) basado en el cambio de especies entre dos muestras, los cuales frecuentemente se utilizan en estudios de diversidad de especies (Magurran, 1989; Moreno, 2001).

\section{RESULTADOS}

Se recolectó un total de 4,232 individuos, que representan 30 géneros y 62 especies, de las cuales 57 especies y 26 géneros pertenecen a Scolytinae y cinco especies y cuatro géneros representan a Platypodinae. Los géneros Hypothenemus y Xyleborus registraron la mayor riqueza de especies con diez y siete respectivamente. La especie Premnobius cavipennis Eichhoff 1878 presentó la mayor abundancia con 741 especímenes lo que equivale al $17.5 \%$ del total (Cuadro 1).

En Malpasito se recolectó la mayor riqueza de especies con 39, sin embargo, la abundancia (363 individuos con el 8.5\%) fue menor con respecto a los otros cuatro sitios de muestreo. Las especies dominantes en esta localidad fueron Stegomerus mexicanus (Wood, 1967), con 178 especímenes (50.57\%), Xyleborus affinis Eichhoff 1968 con 40 (11.3\%) y Corthylus papulans Eichhoff 1869 con 30 (8.26\%); estas especies representan el 70\% de los individuos recolectados en el sitio. 
Cuadro 1. Diversidad de Scolytinae y Platypodinae de cinco localidades de Tabasco, México. (MP Malpasito, BC Boca del Cerro, FR Francisco Rueda, CA Campo Alto, RA Rivera Alta). *Nuevo registro para Tabasco.

\begin{tabular}{|c|c|c|c|c|c|c|c|}
\hline Especies & MP & BC & FR & CA & RA & Total & $\%$ \\
\hline \multicolumn{8}{|l|}{ SCOLYTINAE } \\
\hline Ambrosiodmus hagedorni (Iglesias), 1914 & 0 & 0 & 1 & 0 & 0 & 1 & 0.02 \\
\hline Ambrosiodmus sp. & 0 & 0 & 1 & 0 & 0 & 1 & 0.02 \\
\hline Amphicranus micans Wood, 1976 & 1 & 0 & 0 & 1 & 0 & 2 & 0.05 \\
\hline Araptus tabogae (Blackman), 1942 & 0 & 0 & 0 & 13 & 0 & 13 & 0.31 \\
\hline Cnesinus elegans Blandford, 1896* & 0 & 0 & 0 & 8 & 0 & 8 & 0.19 \\
\hline Cnesinus gracilis Blandford, 1896* & 3 & 1 & 0 & 0 & 0 & 4 & 0.09 \\
\hline Cnesinus sp. & 2 & 0 & 0 & 0 & 0 & 2 & 0.05 \\
\hline Cnesinus squamosus Wood, 1968 & 0 & 0 & 0 & 1 & 0 & 1 & 0.02 \\
\hline Coccotrypes carpophagus (Hornung), 1842 & 3 & 1 & 0 & 0 & 0 & 4 & 0.09 \\
\hline Corthylocurus barbatus (Blandford), 1904* & 1 & 0 & 0 & 0 & 0 & 1 & 0.02 \\
\hline Corthylocurus debilis Wood, 1974 & 20 & 0 & 4 & 11 & 0 & 35 & 0.83 \\
\hline Corthylus flagellifer Blandford, 1904 & 1 & 1 & 0 & 0 & 0 & 2 & 0.05 \\
\hline Corthylus minutissimus Schedl, 1940 & 0 & 0 & 0 & 23 & 0 & 23 & 0.54 \\
\hline Corthylus papulans Eichhoff, 1869 & 30 & 0 & 150 & 93 & 298 & 571 & 13.49 \\
\hline Corthylus sp. & 1 & 1 & 0 & 0 & 0 & 2 & 0.05 \\
\hline Chramesus crenatus Wood, 1956* & 1 & 0 & 0 & 0 & 0 & 1 & 0.02 \\
\hline Cryptocarenus diadematus Eggers, 1937 & 2 & 2 & 0 & 213 & 35 & 252 & 5.95 \\
\hline Cryptocarenus heveae (Hagedorn), 1912 & 2 & 5 & 76 & 41 & 44 & 168 & 3.97 \\
\hline Cryptocarenus lepidus Wood, 1971 & 5 & 3 & 4 & 4 & 0 & 16 & 0.38 \\
\hline Cryptocarenus seriatus Eggers, 1933 & 2 & 2 & 2 & 71 & 60 & 137 & 3.24 \\
\hline Dendrocranulus maurus Wood \& Bright, 1992 & 1 & 0 & 0 & 0 & 0 & 1 & 0.02 \\
\hline Dryocoetoides capucinus (Eichhoff), 1869 & 4 & 5 & 1 & 0 & 0 & 10 & 0.24 \\
\hline Gnathotrupes bituberculatus (Blandford, 1904) & 0 & 0 & 3 & 0 & 0 & 3 & 0.07 \\
\hline Hylocurus effeminatus Wood, 1956* & 0 & 0 & 0 & 1 & 0 & 1 & 0.02 \\
\hline Hylocurus elegans Eichhoff, 1872 & 0 & 0 & 0 & 3 & 0 & 3 & 0.07 \\
\hline Hylocurus inaequalis Wood, 1956* & 0 & 0 & 0 & 1 & 0 & 1 & 0.02 \\
\hline Hypothenemus areccae (Hornung), 1842 & 0 & 0 & 0 & 4 & 2 & 6 & 0.14 \\
\hline Hypothenemus birmanus (Eichhoff), 1878 & 1 & 0 & 3 & 1 & 9 & 14 & 0.33 \\
\hline Hypothenemus brunneus (Hopkins), 1915 & 0 & 0 & 0 & 1 & 0 & 1 & 0.02 \\
\hline Hypothenemus columbi Hopkins, 1915* & 1 & 0 & 2 & 1 & 20 & 24 & 0.57 \\
\hline Hypothenemus crudiae (Panzer), 1791 & 1 & 2 & 0 & 0 & 0 & 3 & 0.07 \\
\hline Hypothenemus dolosus Wood, 1974 & 3 & 10 & 0 & 0 & 0 & 13 & 0.31 \\
\hline Hypothenemus erectus LeConte, 1876 & 1 & 0 & 0 & 0 & 1 & 2 & 0.05 \\
\hline Hypothenemus eruditus Westwood, 1836 & 1 & 2 & 38 & 25 & 3 & 69 & 1.63 \\
\hline Hypothenemus interstitialis (Hopkins), 1915 & 1 & 1 & 461 & 65 & 4 & 532 & 12.57 \\
\hline Hypothenemus seriatus (Eichhoff), 1872 & 0 & 2 & 0 & 1 & 1 & 4 & 0.09 \\
\hline Monarthrum robustum (Schedl), 1966* & 0 & 1 & 0 & 0 & 0 & 1 & 0.02 \\
\hline Pityophthorus sp. & 1 & 0 & 0 & 0 & 0 & 1 & 0.02 \\
\hline Premnobius cavipennis Eichhoff, 1878 & 13 & 7 & 179 & 498 & 44 & 741 & 17.51 \\
\hline Pseudothysanoes tenellus Wood, 1971 & 0 & 0 & 0 & 21 & 3 & 24 & 0.57 \\
\hline
\end{tabular}


Cuadro 1. Continuación...

\begin{tabular}{|c|c|c|c|c|c|c|c|}
\hline Especies & MP & BC & FR & CA & RA & Total & $\%$ \\
\hline Pycnarthrum hispidum (Ferrari), 1867 & 0 & 0 & 0 & 0 & 1 & 1 & 0.02 \\
\hline Sampsonius dampfi Schedl, 1940 & 1 & 529 & 2 & 0 & 1 & 533 & 12.59 \\
\hline Sampsonius mexicanus Bright, 1991 & 1 & 0 & 0 & 0 & 0 & 1 & 0.02 \\
\hline Sampsonius reticulatus Bright, 1972 & 2 & 0 & 0 & 0 & 0 & 2 & 0.05 \\
\hline Stegomerus mexicanus Wood, 1967 & 178 & 5 & 0 & 0 & 1 & 184 & 4.35 \\
\hline Theoborus ricini (Eggers), 1932 & 0 & 0 & 0 & 2 & 1 & 3 & 0.07 \\
\hline Tricolus difodinus Bright, 1972 & 2 & 1 & 0 & 5 & 4 & 12 & 0.28 \\
\hline Xyleborinus gracilis (Eichhoff), 1868 & 0 & 0 & 2 & 0 & 0 & 2 & 0.05 \\
\hline Xyleborus affinis Eichhoff, 1868 & 40 & 0 & 57 & 2 & 0 & 99 & 2.34 \\
\hline Xyleborus ferrugineus (Fabricius), 1801 & 1 & 0 & 7 & 2 & 1 & 11 & 0.26 \\
\hline Xyleborus horridus Eichhoff, 1869 & 1 & 0 & 0 & 2 & 0 & 3 & 0.07 \\
\hline Xyleborus morulus Blandford, 1898 & 0 & 0 & 2 & 0 & 0 & 2 & 0.05 \\
\hline Xyleborus posticus Eichhoff, 1869 & 2 & 0 & 2 & 0 & 0 & 4 & 0.09 \\
\hline Xyleborus spinulosus Blandford, 1898 & 0 & 4 & 5 & 3 & 3 & 15 & 0.35 \\
\hline Xyleborus volvulus (Fabricius), 1775 & 17 & 4 & 485 & 47 & 32 & 585 & 13.82 \\
\hline Xylosandrus curtulus (Eichhoff), 1869 & 0 & 0 & 0 & 0 & 6 & 6 & 0.14 \\
\hline Xylosandrus morigerus (Blandford), 1894 & 1 & 0 & 1 & 0 & 0 & 2 & 0.05 \\
\hline Número de especímenes & 348 & 589 & 1488 & 1164 & 574 & 4163 & \\
\hline Número de especies & 36 & 21 & 23 & 30 & 22 & 57 & \\
\hline \multicolumn{8}{|l|}{ PLATYPODINAE } \\
\hline Euplatypus parallelus (Fabricius), 1801 & 0 & 0 & 1 & 10 & 4 & 15 & 0.35 \\
\hline Euplatypus segnis (Chapuis), 1865 & 5 & 25 & 10 & 0 & 0 & 40 & 0.95 \\
\hline Teloplatypus excisus (Chapuis), 1865 & 9 & 1 & 1 & 0 & 0 & 11 & 0.26 \\
\hline Tesserocerus dewalquei Chapuis, 1865 & 1 & 0 & 0 & 0 & 0 & 1 & 0.02 \\
\hline Platyphysus obtusus (Chapuis), 1865 & 0 & 2 & 0 & 0 & 0 & 2 & 0.05 \\
\hline Número de especímenes & 15 & 28 & 12 & 10 & 4 & 69 & \\
\hline Número de especies & 3 & 3 & 3 & 1 & 1 & 5 & \\
\hline Total de especímenes & 363 & 617 & 1500 & 1174 & 578 & 4232 & \\
\hline Total de especies & 39 & 24 & 26 & 31 & 23 & 62 & \\
\hline
\end{tabular}

En Boca del Cerro se capturaron 24 especies con un total de 617 especímenes (14.58\%). La especie Sampsonius dampfi Schedl 1940 fue la dominante en esta zona con 529 organismos, lo que representó el 86.58\% de la abundancia.

En Francisco Rueda se registró la máxima abundancia con respecto a los otros sitios muestreados, recolectando un total de 1,500 (35.44\%) organismos de 26 especies. Las especies con mayor abundancia fueron Xyleborus volvulus (Fabricius) 1775, con 485 especímenes (32.48\%), seguido de Hypothenemus interstitialis (Hopkins) 1915 con 461 (30.88\%), P. cavipennis con 179 (11.99\%) y C. papulans con 150 (10.0\%), estas especies representan el $85.40 \%$ de la abundancia recolectada en este sitio.

Para Campo Alto, se identificaron 31 especies, sumando un total de 1,174 individuos (27.74\% del total). En esta localidad, la especie con más incidencia fue Premnobius cavipennis con 498 especímenes (43.88\%), seguida por Cryptocarenus diadematus Eggers 1937 con 213 (18.77\%). 
Para el caso de Rivera Alta, la riqueza fue de 23 especies, con una abundancia de 578 especímenes (13.66\%). En este sitio la especie dominante fue Corthylus papulans con 297 organismos (53.80\%), seguida de Cryptocarenus seriatus Eggers 1933 con 60 (10.87\%).

Las especies Ambrosiodmus hagedorni (Iglesias), 1914, Cnesinus squamosus Wood, 1968, Corthylocurus barbatus (Blandford), 1904, Chramesus crenatus Wood, 1956, Dendrocranulus maurus Wood \& Bright, 1992, Hylocurus effeminatus Wood, 1956, H. inaequalis Wood, 1956, Hypothenemus brunneus (Hopkins), 1915, Monarthrum robustum (Schedl), 1966, Pycnarthrum hispidum (Ferrari), 1867, Sampsonius mexicanus Bright, 1991 y Tesserocerus dewalquei Chapuis, 1865 estuvieron representadas por un solo ejemplar.

De acuerdo con el índice de Shannon-Wiener $\left(\mathrm{H}^{\prime}\right)$, la máxima diversidad se presentó en MP (2.11) y la mínima en BC (0.78). Respecto al índice de equidad (J), CA presentó la máxima (0.59), mientras que BC obtuvo la mínima (0.25). El índice de Margalef (Dmg) registró que MP fue la localidad con la máxima riqueza específica (Cuadro 2). La prueba estadística de $t\left(t_{0.05}=1.96\right)$ aplicada a la diversidad $\left(\mathrm{H}^{\prime}\right)$ de Scolytinae y Platypodinae demostró que existen diferencias significativas entre los sitios muestreados, con excepción de MP-CA y FR-RA. El índice de similitud de Sorensen (Is) determinó que MP-BC y CA-RA son sitios similares debido a que tienden a compartir el mayor número de especies (Cuadro 3).

Cuadro 2. Análisis de la diversidad de Scolytinae y Platypodinae capturados en localidades de Tabasco. (MP Malpasito, BC Boca del Cerro, FR Francisco Rueda, CA Campo Alto, RA Rivera Alta).

\begin{tabular}{ccccccc}
\hline Localidades & Especies (S) & Abundancia (N) & Diversidad (H') & Equidad (J) & Var. $\mathbf{H}^{\prime}$ & Riqueza (Dmg) \\
\hline MP & 39 & 363 & 2.11 & 0.58 & 0.00724 & 6.446 \\
BC & 24 & 617 & 0.78 & 0.25 & 0.00409 & 3.579 \\
FR & 26 & 1500 & 1.8 & 0.55 & 0.0008 & 3.418 \\
CA & 31 & 1174 & 2.03 & 0.59 & 0.00154 & 4.244 \\
RA & 23 & 578 & 1.81 & 0.58 & 0.0032 & 3.459 \\
\hline
\end{tabular}

Cuadro 3. Comparaciones de índice de similitud de Sorensen (Is) de Scolytinae y Platypodinae capturados en localidades de Tabasco. Valores indicados por asterisco no presentan diferencia significativa $\left(t_{0.05}\right)$. (MP Malpasito, BC Boca del Cerro, FR Francisco Rueda, CA Campo Alto, RA Rivera Alta).

\begin{tabular}{ccccc}
\hline Localidades & MP & BC & FR & CA \\
\hline MP & - & - & - & - \\
BC & 0.635 & - & - & - \\
FR & 0.585 & 0.480 & - & - \\
CA & $0.486^{*}$ & 0.400 & 0.526 & - \\
RA & 0.484 & 0.511 & $0.531^{*}$ & 0.667 \\
\hline
\end{tabular}

\section{DISCUSIÓN}

En el estado de Tabasco existen particularidades biogeográficas que determinan la estructura de las comunidades de Scolytinae y Platypodinae, mostrando una composición de especie variable en cada tipo 
de vegetación de acuerdo con los estudios que realizaron Pérez-De La Cruz et al. (2009; 2015; 2016) y Gerónimo-Torres et al. (2015), éste estudio permitió incrementar el conocimiento de la diversidad y distribución de estos grupos, reportando ocho nuevos registros para el estado. La diversidad de Scolytinae y Platypodinae, presentó de manera general, variaciones tanto en composición como en abundancia entre sitios, algo similar a lo que se ha reportado en estudios previos (Pérez-De La Cruz et al., 2009; 2015; Gerónimo-Torres et al., 2015). El índice de diversidad y similitud indicaron que existen diferencias en la riqueza de especies entre la mayoría de las localidades analizadas, estas diferencias podrían estar dadas por los elementos que componen cada ambiente, debido a que los factores abióticos como temperatura y humedad, y bióticos como estructura de la vegetación, disponibilidad de alimento, interacciones de depredación, competencia, así como, sus interacciones son quienes determinan la diversidad y abundancia de estos insectos (Rudinsky, 1962; Wood, 1982; Pérez-De La Cruz et al., 2009).

Los índices de diversidad permitieron comparar la composición de especies encontrada en los sitios de estudio, registrando a MP como el más diverso, siendo un área de selva conservada con abundante vegetación, a diferencia de FR que presentó la mayor abundancia siendo un monocultivo de $H$. brasiliensis, aunado a RA donde la vegetación leñosa es limitada, estos dos últimos sitios registraron baja riqueza de especie, lo que sugiere que la pérdida de vegetación contribuye a la pérdida de la diversidad de estos insectos, así como, se favorece el establecimiento e incremento de las poblaciones de algunas especies de insectos generalistas, tal como se observó con Hypothenemus interstitialis y Xyleborus volvulus en FR.

Las especies Cnesinus elegans, C. gracilis, Corthylocurus barbatus, Chramesus crenatus, Hylocurus effeminatus, $H$. inaequalis, Hypothenemus columbi, y Monarthrum robustum son nuevos registros para Tabasco, sumando un total de 101 especies de Scolytinae con base a lo registrado anteriormente (Schedl, 1940; Equihua \& Burgos, 2002; Pérez-De La Cruz et al., 2009; 2015). Los géneros Hypothenemus y Xyleborus presentaron la mayor riqueza de especies con 10 cada uno. Resultados similares han sido reportados en plantaciones de cacao (Theobroma cacao L.), manglares y selvas tropicales de México ya que ambos géneros son de amplia distribución y su mayor diversidad se ha registrado en regiones tropicales y subtropicales del mundo (Atkinson \& Equihua, 1986; Pérez-De la Cruz et al., 2009; 2015).

Las especies Xyleborus affinis, $X$. ferrugineus y $X$. volvulus están reportadas como especies generalistas con amplia distribución y una significativa ocurrencia en el agroecosistema de cacao, selvas, manglares y vegetación secundaria de Tabasco. Además, la distribución geográfica de estas tres especies va más allá de los límites tropicales, inclusive de nuestro país, alcanzando localidades que pertenecen a la región neártica del continente (Wood \& Bright, 1992a). En este estudio no fueron las especies más abundante a excepción de $X$. volvulus que fue la segunda especie con mayor abundancia, la cual es considerada de importancia forestal en las áreas tropicales (Cibrián-Tovar et al., 1995), debido a que esta especie tiene como huésped a 231 especies de plantas que están incluidas en 28 familias, lo que le permite alcanzar altas densidades poblacionales por la gran disponibilidad de alimento.

La alta abundancia de Premnobius cavipennis es algo novedoso, ya que en selvas y manglares ha sido reportada con una baja abundancia (Pérez-De La Cruz et al., 2015; Gerónimo-Torres et al., 2015), sin embargo, en plantaciones de cacao es una especie donde sus poblaciones han incrementado (Pérez-De La Cruz et al., 2009), está reportada en África y tiene una amplia distribución en el continente americano con una amplia variedad de huéspedes (Wood \& Bright, 1992a; 1992b).

La lista de especies de estos insectos en Tabasco, México continúa creciendo conforme se realizan nuevos muestreos, lo que hace pensar que no se ha logrado recolectar la diversidad real que albergan los ecosistemas de Tabasco. Además, los estados vecinos de Veracruz (274 especies) y Chiapas (170) tienen 
registrado un número de especies superior a lo que se ha recolectado en el estado (Equihua \& Burgos, 2002).

Las trampas de alcohol son recomendables para la captura de insectos barrenadores de madera (Scolytinae y Platypodinae), sin embargo, es común recolectar insectos ambrosiales (diversidad alta en el trópico) en mayor medida con respecto a otros Scolytinae con diferentes hábitos alimenticios. Por lo tanto, es recomendable utilizar métodos de recolecta complementarios (captura directa sobre sus plantas huésped, trampas de luz o uso de semioquímicos) que nos permitan acercarnos en lo posible al número real de especies que albergan nuestros ecosistemas. A pesar de que el número de especies aquí reportados es similar a lo registrado por Estrada \& Atkinson (1988) para Campeche y menor a los reportado por Atkinson \& Equihua (1986) para selvas de una región de Veracruz y Oaxaca, en donde utilizaron la captura directa sobre sus plantas huésped, el número de especies aquí reportado es significativo, dado que el tiempo y los recursos empleados son limitantes.

Es necesario continuar con el monitoreo de estas especies dado que actualmente es común que se introduzcan nuevas especies, las cuales pueden causar mayores daños, tales como; Xyleborus glabratus (Eichhoff) que es uno de los insectos barrenadores de madera más destructivos en Norte América, asociado a la marchitez del laurel, causado por Raffaelea lauricola (Fraedrich, 2008; Harrington et al., 2010) y Euwallacea fornicatus (Eichhoff) que es considerada plaga y que actualmente fue introducida a California y Centro América y afecta a plantas de Persea americana Mill (Kirkendall \& Odegaard, 2007; Freeman et al., 2013; Smith \& Hulcr, 2014). Estas especies han causado daños en diferentes ecosistemas y actualmente están próximas de entrar a territorio mexicano donde se distribuyen sus plantas huéspedes. Por lo tanto, es importante que se mantengan estudios que coadyuven al monitoreo de estas especies.

AgRAdECIMIENTOS. El primer autor agradece al Consejo Nacional de Ciencia y Tecnología (CONACYT), por el apoyo económico para el estudio de Maestría en Ciencias ambientales de la Universidad Juárez Autónoma de Tabasco, México, tiempo durante el cual desarrollé esta investigación. Así como, a los revisores por las observaciones y aportaciones realizadas al manuscrito.

\section{LITERATURA CITADA}

Atkinson, T. H., Equihua, M. A. (1986) Biology of bark and ambrosia beetles (Coleoptera: Scolytidae and Platypodidae) of a tropical rain forest in southeastern Mexico with an annotated checklist of Species. Annals of the Entomological Society of America, 79, 414-423.

Bustamante, O. F., Atkinson T. H. (1984) Biología del barrenador de las ramas del peral Corthylus fuscus Blandford (Coleoptera: Scolytidae), en el norte del Estado de Morelos. Folia Entomológica Mexicana, 60, 83-101.

Cibrián-Tovar, D., Méndez-Montiel, J. T., Campos-Bolaños, R., Yates III, H. O., Flores-Lara, J. E. (1995) Insectos forestales de México. UACH. Sec. Agr. Recursos Hidráulicos, México. USDA, For. Serv., Nat. Resources, Canadá, Com. For. De América del Norte, FAO. Chapingo, México, $453 \mathrm{pp}$.

Equihua, M. A., Burgos, S. A. (2002) Scolytidae. Pp. 539-557. In: J. Llorente-Bousquets \& J. J. Morrone (Eds). Biodiversidad, Taxonomía y Biogeografía de artrópodos de México: Hacia una síntesis de su conocimiento. Vol. III. CONABIO-IBUNAM. México.

Estrada, V. A., Atkinson T. H. (1988) Scolytidae y Platypodidae (Coleoptera) de Escárcega, Campeche, México. Biogeografía, Biología, importancia económica y una lista comentada de especies. Anales del Instituto de Biología Universidad Nacional Autónoma de México. Serie Zoología, 1, 199-220.

Fraedrich, S. W. (2008) California laurel is susceptible to laurel wilt caused by Raffaelea lauricola. Plant Disease, 92, 1469. 
Freeman, S., Sharon, M., Maymon, M., Mendel, Z., Protasov, A., Aoki, T. (2013) Fusarium euwallaceae sp. nov., a symbiotic fungus of Euwallacea sp., an invasive ambrosia beetle in Israel and California. Mycologia, 105, 1595-1606.

Gerónimo-Torres, J. C., Pérez-De La Cruz, M., De La Cruz-Pérez, A., Torres-De La Cruz, M. (2015) Scolytinae y Platypodinae (Coleoptera: Curculionidae) asociados a manglares de Tabasco, México. Revista Colombiana de Entomología, 41(2), 257-261.

Guzmán A. C. C., Bello G. J. (2006) Mamíferos de Boca del Cerro Tenosique, Tabasco, México. Kuxulkab, 11, 75-84.

Haack, R. A. (2006) Exotic bark and wood-boring Coleoptera in the United States: recent establishments and interceptions. Canadian Journal of Forestry Research, 36, 269-288.

Harrington, T. C., Aghayeva, D. N. Fraedrich, S. W. (2010) New combinations in Raffaelea, Ambrosiella, and Hyalorhinocladiella, and four new species from the redbay ambrosia beetle, Xyleborus glabratus. Mycotaxon, 111, 337-361.

INEGI (Instituto Nacional de Estadística Geografía e Informática) (2000) Cuaderno estadístico de Tenosique, Tabasco. Gobierno del Estado de Tabasco, 44 pp.

INEGI (Instituto Nacional de Estadística Geografía e Informática) (2001) Carta topográfica, Tenosique, Tabasco. E15D35. Esc. 1:50 000.

INEGI (Instituto Nacional de Estadística Geografía e Informática) (2013) Mapa digital de elevación Vol. V. <http://gaia.inegi.org.mx/mdm6/> (Consultado en Marzo de 2014).

Iturre, M., Darchuck E. (1996) Registros de escolítidos relacionados al género Eucalyptus en Santiago del Estero. Instituto de control biológico, Facultad de Ciencias Forestales. Universidad Nacional de Santiago del Estero. Argentina. Quebracho, 4, 11-19.

Kirkendall, L. R., Odegaard F. (2007) Ongoing invasions of old-growth tropical forests: Establishment of three incestuous beetle species in Central America (Curculionidae, Scolytinae). Zootaxa, 1588, 53-62.

Kirkendall, L. R., Faccoli M. (2010) Bark beetles in pinhole borers (Curculionidae, Scolytinae, Platypodinae) alien to Europe. ZooKeys, 56, 227-251.

Kirkendal, L. R., Biedermann P. H. W., Jordal B. H. (2014) Evolution and Diversity of Bark and Ambrosia Beetles. Pp. 85-156. In: F. E. Vega \& R. W. Hofstetter (Eds). Bark beetles, biology and ecology of native and invasive species. United States. Academic Press, London and San Diego.

Logan, J. A., Powell J. A. (2001) Ghost Forest, Global warming, and the mountain pine beetle (Coleoptera: Scolytidae). American Entomologist, 47, 160-173.

Magurran, E. A. (1989) Diversidad ecológica y su medición. Ed. Vedra. ISBN. 84-87456-00-6. Barcelona, España, 200 pp.

Moreno, C. E. (2001) Métodos para medir la biodiversidad. M\&T. ORCYT/UNESCO/SEA (Ed.). Primera edición. Vol. 1. Zaragoza, España, 83 pp.

Pérez-De La Cruz, M., Equihua-Martínez A., Romero-Nápoles J., Sánchez-Soto S., García-López E. (2009) Diversidad, fluctuación poblacional y plantas huésped de escolitinos (Coleoptera: Curculionidae) asociados con el agroecosistema cacao en Tabasco, México. Revista Mexicana de Biodiversidad, 80, 779-791.

Pérez-De La Cruz M, Valdez-Carrasco J., M, Romero-Nápoles J., Equihua-Martínez A., SánchezSoto S., De La Cruz-Pérez A. (2011) Fluctuación poblacional, plantas huéspedes, distribución y clave para la identificación de Platypodinae (Coleoptera: Curculionidae) asociados al agroecosistema cacao en Tabasco, México. Acta Zoológica Mexicana (n.s), 27, 129-143.

Pérez-De la Cruz, M., Zavaleta-Bastar, P. G., De la Cruz-Pérez A. (2015) Aproximación al conocimiento de la diversidad de Scolytinae y Platypodinae (Coleoptera: Curculionidae) asociados a selvas de Tabasco, México. Entomotropica, 30(20), 201-211.

Pérez-De la Cruz, M., Hernández-May, M. A., De la Cruz-Pérez, A., Sánchez-Soto, S. (2016) Scolytinae y Platypodinae (Coleoptera: Curculionidae) de dos áreas de conservación en Tabasco, México. Revista de Biología Tropical/International Journal of Tropical Biology and Conservation, 64(1), 319-326. 
Triplehorn, C. A., Johnson N. F. (2005) An introduction to the study of insects. $7^{\text {th }}$ Ed. Philadelphia, Saunders College Publishing, 865 pp.

Rudinsky, L. (1962) Ecology of Scolytidae. Annual Review of Entomology, 7, 327-348.

Schedl, K. E. (1940) Scolytidae, Coptonotidae y Platypodidae mexicanos. Anales de la Escuela Nacional de Ciencias Biológicas, 1, 317-376.

Smith, S. M., Hulcr J. (2015) Scolytus and other economically important bark and ambrosia beetles. Pp. 495-532. In: F. E. Vega \& R. W. Hofstetter (Eds). Bark beetles, biology and ecology of native and invasive species. United States. Academic Press, London and San Diego.

Wood, S. L. (1982) The bark and ambrosia beetles of North and Central America (Coleoptera: Scolytidae), a taxonomic monograph. Great Basin Naturalist Memoirs, 6, 1-1327.

Wood, S. L. (1993) Revision of the genera of Platypodidae (Coleoptera). Great Basin Naturalist, 53, 259281.

Wood, S. L., Bright D. E. (1992a) A catalog of Scolytidae and Platypodidae (Coleoptera), Part 2. Taxonomic Index. Great Basin Naturalist Memoirs, 13, 1-833.

Wood, S. L., Bright D. E. (1992b) A catalog of Scolytidae and Platypodidae (Coleoptera), Part 2. Taxonomic Index. Great Basin Naturalist Memoirs, 13, 835-1553. 